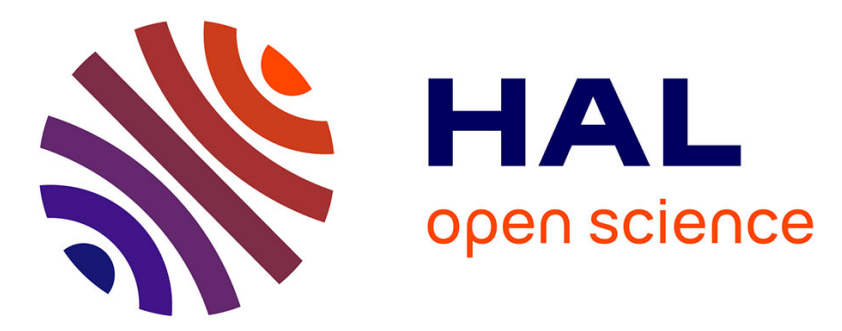

\title{
More light on the $2 \nu 5$ Raman overtone of SF6: Can a weak anisotropic spectrum be due to a strong transition anisotropy?
}

David Kremer, Florent Rachet, Michel Chrysos

\section{- To cite this version:}

David Kremer, Florent Rachet, Michel Chrysos. More light on the $2 \nu 5$ Raman overtone of SF6: Can a weak anisotropic spectrum be due to a strong transition anisotropy?. Journal of Chemical Physics, 2014, 140 (3), pp.034308. 10.1063/1.4861047 . hal-03344837

\section{HAL Id: hal-03344837 \\ https://univ-angers.hal.science/hal-03344837}

Submitted on 15 Sep 2021

HAL is a multi-disciplinary open access archive for the deposit and dissemination of scientific research documents, whether they are published or not. The documents may come from teaching and research institutions in France or abroad, or from public or private research centers.
L'archive ouverte pluridisciplinaire HAL, est destinée au dépôt et à la diffusion de documents scientifiques de niveau recherche, publiés ou non, émanant des établissements d'enseignement et de recherche français ou étrangers, des laboratoires publics ou privés. 


\section{AD $\mid \begin{aligned} & \text { The Journal of } \\ & \text { Chemical Physics }\end{aligned}$}

More light on the 2v5 Raman overtone of SF6: Can a weak anisotropic spectrum be due to a strong transition anisotropy?

D. Kremer, F. Rachet, and M. Chrysos

Citation: The Journal of Chemical Physics 140, 034308 (2014); doi: 10.1063/1.4861047

View online: http://dx.doi.org/10.1063/1.4861047

View Table of Contents: http://scitation.aip.org/content/aip/journal/jcp/140/3?ver=pdfcov

Published by the AIP Publishing

\section{Articles you may be interested in}

On the isotropic Raman spectrum of Ar2 and how to benchmark ab initio calculations of small atomic clusters:

Paradox lost

J. Chem. Phys. 143, 024304 (2015); 10.1063/1.4923370

From light-scattering measurements to polarizability derivatives in vibrational Raman spectroscopy: The 2v5 overtone of SF6

J. Chem. Phys. 138, 174308 (2013); 10.1063/1.4803160

The isotropic remnant of the CO2 near-fully depolarized Raman 2v3 overtone

J. Chem. Phys. 134, 104310 (2011); 10.1063/1.3557820

Isotropic and anisotropic collision-induced light scattering by gaseous sulfur hexafluoride at the frequency region of the $v 1$ vibrational Raman line

J. Chem. Phys. 118, 11009 (2003); 10.1063/1.1575733

Isotropic collision induced light scattering spectra from gaseous SF 6

J. Chem. Phys. 116, 5337 (2002); 10.1063/1.1463421

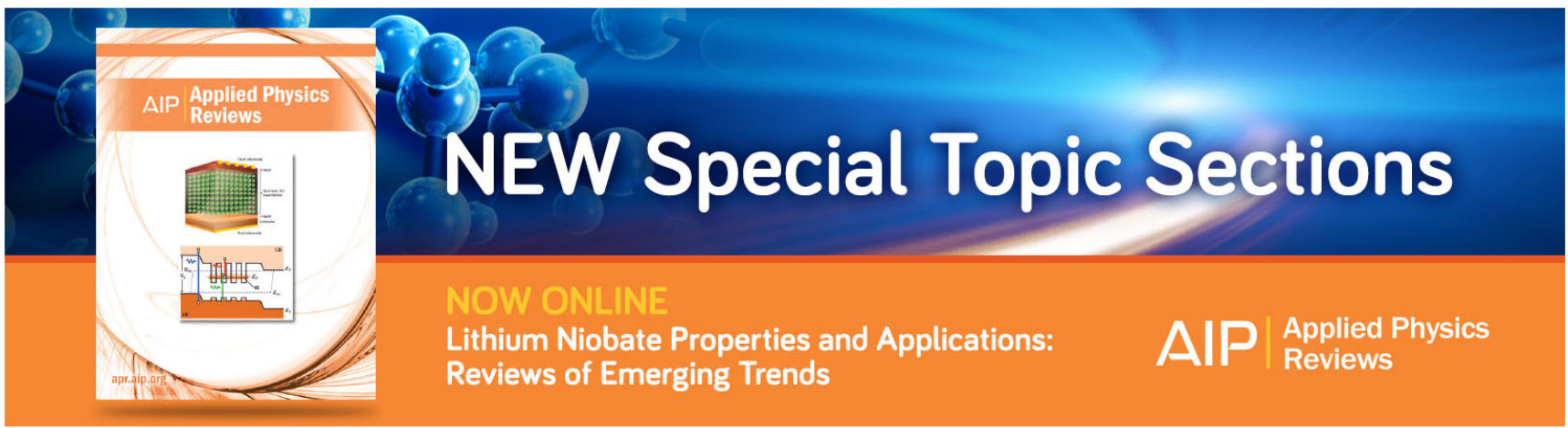




\title{
More light on the $2 v_{5}$ Raman overtone of $\mathrm{SF}_{6}$ : Can a weak anisotropic spectrum be due to a strong transition anisotropy?
}

\author{
D. Kremer, F. Rachet, and M. Chrysos ${ }^{\text {a) }}$ \\ LUNAM Université, Université d'Angers, CNRS UMR 6200, Laboratoire MOLTECH-Anjou, 2 Bd Lavoisier, \\ 49045 Angers, France
}

(Received 24 November 2013; accepted 16 December 2013; published online 16 January 2014)

\begin{abstract}
Long known as a fully polarized band with a near vanishing depolarization ratio $\left[\eta_{s}=0.05, \mathrm{~W}\right.$. Holzer and R. Ouillon, Chem. Phys. Lett. 24, 589 (1974)], the $2 v_{5}$ Raman overtone of $\mathrm{SF}_{6}$ has so far been considered as of having a prohibitively weak anisotropic spectrum [D. P. Shelton and L. Ulivi, J. Chem. Phys. 89, 149 (1988)]. Here, we report the first anisotropic spectrum of this overtone, at room temperature and for 13 gas densities ranging between 2 and 27 amagat. This spectrum is 10 times broader and 50 times weaker than the isotropic counterpart of the overtone [D. Kremer, F. Rachet, and M. Chrysos, J. Chem. Phys. 138, 174308 (2013)] and its profile much more sensitive to pressure effects than the profile of the isotropic spectrum. From our measurements an accurate value for the anisotropy matrix-element $|\langle 000020|\Delta \alpha| 000000\rangle|$ was derived and this value was found to be comparable to that of the mean-polarizability $|\langle 000020|\bar{\alpha}| 000000\rangle|$. Among other conclusions our study offers compelling evidence that, in Raman spectroscopy, highly polarized bands or tiny depolarization ratios are not necessarily incompatible with large polarizability anisotropy transition matrix-elements. Our findings and the way to analyze them suggest that new strategies should be developed on the basis of the complementarity inherent in independent incoherent Raman experiments that run with two different incident-beam polarizations, and on concerted efforts to $a b$ initio calculate accurate data for first and second polarizability derivatives. Values for these derivatives are still rarities in the literature of $\mathrm{SF}_{6}$. $\odot 2014$ AIP Publishing LLC. [http://dx.doi.org/10.1063/1.4861047]
\end{abstract}

\section{INTRODUCTION}

Raman spectroscopy in gases is an invaluable tool for scientists to delve deeper into the inner workings of matter. ${ }^{1}$ Even for particles with relatively simple structure (atoms, simple molecules), the Raman effect can reveal a wealth of information about complex (often poorly understood or unknown) polarization mechanisms, which operate during the vibrational motion of the gas molecules ${ }^{2,3}$ or, transiently, as two or several gas particles happen to come close to each other in the course of their random movement ("collision"). ${ }^{4-7}$ Although most of these unfamiliar mechanisms have hardly perceptible Raman effects, their physical significance is huge and, in spite of the great attention Raman spectroscopy has been receiving for decades, there are still new and intriguing processes to uncover provided that reliable Raman spectra come to spot them with high-sensitivity experiments. ${ }^{8-14}$ Of the possible vibrations in a polyatomic molecule, pure bending ones are among the most difficult to describe spectroscopically for they deform the molecule without distorting its chemical bonds. Sulphur hexafluoride has been witnessing a revival of spectroscopic and computational interest in the last two decades. ${ }^{8-10,15-19}$ This molecule belongs to the octahedral symmetry group and its $v_{5}$ mode is the par excellence bending vibration. In this vibration, four fluorine atoms in a plane undergo "scissoring" motion about the molecular center with the remaining three atoms being at rest on an axis perpendicular to the plane. ${ }^{20}$ The quantitative knowledge of several

a)Electronic mail: michel.chrysos@univ-angers.fr spectroscopic parameters related to the $v_{5}$ transition of $\mathrm{SF}_{6}$ (polarizability derivatives, cubic force constants, etc.) is still Terra incognita for chemical physics, even though it has long been known that $v_{5}$ is allowed in Raman spectroscopy and that its spectrum is fully depolarized. Going to a higher level of complexity, it has been concluded from Raman experiments that the $2 v_{5}$ overtone (which is also allowed in Raman spectroscopy) generates a highly polarized band spectrum with a depolarization ratio that is almost vanishingly small. ${ }^{21,22}$ In a recent article, highly accurate isotropic spectra have been reported for this overtone and a systematic procedure to determine second derivatives for the mean dipole polarizability of this vibration was developed. ${ }^{23}$ Such quantities are of a significant physical content and their contribution to the understanding of the "greenhouse mechanisms" can be substantial. Yet owing to the difficulty of making ab initio computations for those quantities, values of derivatives other than for the "breathing" $v_{1}$ mode $^{16}$ or reliable values for the cubic force constants, especially for the bending modes of $\mathrm{SF}_{6}$, are rarities in the literature of this molecule.

In the present paper, we report the first-ever anisotropic spectrum of this overtone. This spectrum, which is ten times broader than its predominant isotropic counterpart, turns out to peak two orders of magnitude below the maximum of the isotropic overtone component. Accurate values for the anisotropic zeroth- and second-order spectral moments, $M_{0}, M_{2}$, and for the polarizability anisotropy matrix-element $\langle 000020|\Delta \alpha| 000000\rangle$ of this overtone were deduced and are reported below. Surprisingly, in spite of the overall weak intensity of the anisotropic spectrum, $I_{a n i}(v)$, as compared 
to the isotropic counterpart, $I_{\text {iso }}(v)$, the zeroth-order spectral moments, $M_{0}$, of these two spectra were found to take comparable values. So too was the case with the value of $\langle 000020|\Delta \alpha| 000000\rangle$ upon comparison with the recently reported $^{23}\langle 000020|\bar{\alpha}| 000000\rangle$. In light of these findings, the situation of a highly polarized transition $f \leftarrow i$ whose matrixelement $(\Delta \alpha)_{f i}$ is as strong as $(\bar{\alpha})_{f i}$ appears as a rare exception, and to the best of our knowledge has never been encountered before in the vibrational spectroscopy of $\mathrm{SF}_{6}$.

\section{HISTORICAL BACKGROUND AND THE STATE OF THE ART}

The first Raman measurements of $\mathrm{SF}_{6}$ spectra and their rather exhaustive listing of band strengths and widths for a multitude of fundamental, overtone, and combination bands go back to the pioneering works by Shelton and Ulivi. ${ }^{22}$ But as far as the specific $2 v_{5}$ overtone is concerned, the first study of that band dates back even earlier, to a work by Holzer and Ouillon. ${ }^{21}$ In both those works, a fixed polarization perpendicular $(\perp)$ to the scattering plane had been used for the incident beam, which had unavoidably restricted the recorded spectra to their polarized $I_{\perp}$ components. ${ }^{21,22}$ Polarized components by no means are sufficient in a Raman treatment, unless for a strictly polarized band. Even in the presence of an analyzer crossing the scattering beam and switched at will to be set parallel or perpendicular to the scattering plane, the so recorded $I^{(\perp \|)}$ and $I^{(\perp \perp)}$ scattering spectra $\left(I_{\perp}=I^{(\perp \|)}\right.$ $\left.+I^{(\perp \perp)}\right),{ }^{24}$ respectively, are not accurate enough, in principle, to allow for a reliable measurement of both the physical quantities $\langle 000020|\bar{\alpha}| 000000\rangle$ and $\langle 000020|\Delta \alpha| 000000\rangle$. Only with the use of two independent polarizations for the incident beam in running independent Raman experiments can those two quantities be determined safely, and our group has developed, since more than a decade ago, protocols following such a strategy to increase reliability. ${ }^{25-29}$

While the polarized $I_{\perp}$ component of the $2 v_{5} \mathrm{SF}_{6}$ overtone, treated in the previous works, ${ }^{21,22}$ can to some extent be considered as a rather reasonable approximation of the overtone's isotropic spectrum, the latter spectrum had to await, for its rigorous analysis, a study made recently by our group. ${ }^{23}$ In contrast, none of the previous treatments has ever mentioned anything about some anisotropic spectrum for that overtone. This is exactly what the present article focuses on, following a systematic observation and its analysis to fill the gap in the existing literature.

\section{THEORETICAL: SYMMETRIES AND REPRESENTATIONS}

The $2 v_{5}$ overtone corresponds to a reducible representation, which is the symmetrized square of $F_{2 g}$

$$
F_{2 g} \otimes F_{2 g}=A_{1 g}\left(v_{1}\right) \oplus E_{g}\left(v_{2}\right) \oplus F_{2 g}\left(v_{5}\right)
$$

The above expansion coincides with the polarizability representation $\Gamma_{\alpha}$, the latter being built on the basis of the polariz- ability components ${ }^{1}$

$$
\begin{aligned}
& A_{1 g}: \alpha_{x x}+\alpha_{y y}+\alpha_{z z}, \\
& E_{g}: \alpha_{x x}+\alpha_{y y}-2 \alpha_{z z}, \alpha_{x x}-\alpha_{y y}, \\
& F_{2 g}: \alpha_{x y}, \alpha_{y z}, \alpha_{z x} .
\end{aligned}
$$

Whereas the isotropic component of the $2 v_{5}$ band is given by the trace of $\alpha$ and corresponds to the $A_{1 g}$ polarizability component, the anisotropic component of the $2 v_{5}$ band is generated by the anisotropy of $\alpha$. In this respect, $\alpha_{x x}+\alpha_{y y}$ $-2 \alpha_{z z}$ and $\alpha_{x x}-\alpha_{y y}$ determine the overtone transition from the ground state to the $E_{g}$ sublevel of $2 \nu_{5}$ and $\alpha_{x y}, \alpha_{y z}$, $\alpha_{z x}$ determine the overtone transition from the ground state to the $F_{2 g}$ sublevel. Note that the three sublevels $A_{1 g}, E_{g}$, and $F_{2 g}$ are mechanically coupled to the three fundamental "gerade" levels $v_{1}, v_{2}$, and $v_{5}$, respectively, obeying the restrictions $A_{1 g} \Leftrightarrow A_{1 g}, E_{g} \Leftrightarrow E_{g}, F_{2 g} \Leftrightarrow F_{2 g}$ because the coupling potential is always of $A_{1 g}$ symmetry. There are also symmetry restrictions for the polarizability overtone couplings to the matrix-elements of the fundamental vibrations, i.e., to the first polarizability derivatives. The equations also contain the perturbation matrix-elements of the cubic anharmonic terms which couple $A_{1 g}\left(2 v_{5}\right)$ to $A_{1 g}\left(v_{1}\right), E_{g}\left(2 v_{5}\right)$ to $E_{g}\left(v_{2}\right)$, and $F_{2 g}\left(2 v_{5}\right)$ to $F_{2 g}\left(v_{5}\right)$. The cross couplings are forbidden. The same holds true for the components $\alpha\left(A_{1 g}\left(2 v_{5}\right)\right), \alpha\left(E_{g}\left(2 v_{5}\right)\right)$, and $\alpha\left(F_{2 g}\left(2 v_{5}\right)\right)$, which are merely expressed as a function of $\alpha\left(A_{1 g}\left(v_{1}\right)\right), \alpha\left(E_{g}\left(v_{2}\right)\right)$, and $\alpha\left(F_{2 g}\left(v_{5}\right)\right)$, respectively, i.e., as a function of the corresponding derivatives $\partial \alpha / \partial q_{1}, \partial \alpha / \partial q_{2}$, and $\partial \alpha / \partial q_{5}$. This result greatly simplifies the analysis of the Raman spectrum, but does not allow for distinguishing between the two components $\alpha\left(E_{g}\left(2 v_{5}\right)\right)$ and $\alpha\left(F_{2 g}\left(2 v_{5}\right)\right)$ of the anisotropic spectrum (see Ref. 22, p. 151).

It is inferred from the above that the anisotropic spectrum of the overtone comes from a mixture of two $\Delta \alpha$ components: $\alpha_{x x}-\alpha_{y y}$ and $\alpha_{x y}$. If the intensity of the overtone were to lie entirely with one of those channels, either of the two expressions would be true: $(\Delta \alpha)_{f i}^{2}=\frac{3}{2}\langle 000020| \alpha_{x x}$ $-\alpha_{y y}|000000\rangle^{2}$ for $E_{g}$ or $(\Delta \alpha)_{f i}^{2}=9\left\langle 000020\left|\alpha_{x y}\right| 000000\right\rangle^{2}$ for $F_{2 g}$, when summed over degeneracy. Yet, the way in which the total intensity of the anisotropic spectrum is shared between the two channels cannot be deduced experimentally. Only upper bounds are given below for the quantities $\left\langle 000020\left|\alpha_{x y}\right| 000000\right\rangle$ and $\left\langle 000020\left|\alpha_{x x}-\alpha_{y y}\right| 000000\right\rangle$, assuming that either the $E_{g}$ or $F_{2 g}$ species could give the dominant contribution to $(\Delta \alpha)_{f i}^{2}$.

Upon deriving $(\Delta \alpha)_{f i}^{2}$ from the Raman intensity, proper account of the hotband $\gamma_{i}(T)$-factor ${ }^{23}$ is all we need $\left[\gamma_{5}(294 \mathrm{~K})=1.17\right]$. No explicit mention to the internal structure of $(\Delta \alpha)_{f i}$ is necessary. The latter is a rather complicated combination of second harmonic derivatives of the two channel invariants $\left(\alpha_{x x}-\alpha_{y y}\right)$ and $\alpha_{x y}$ with respect to the threefold degenerated normal coordinate $q_{5}$, augmented by anharmonic corrections that involve cubic force constants ${ }^{30} k_{555}\left(=\frac{\phi_{555}}{3 !}\right)$ and $k_{255}\left(=\frac{\phi_{255}}{2 !}\right)$ along with first harmonic derivatives with respect to $q_{5}$ and $q_{2}$. No data are available for any of the aforementioned direct or cross second derivatives of $\left(\alpha_{x x}-\alpha_{y y}\right)$ or $\alpha_{x y}$ while the known values for the constant $\phi_{255}\left(=\sqrt{\frac{8}{3}} C_{255}\right)$ are either unreliable or inconsistent $\left(C_{255}=18.4 \mathrm{~cm}^{-1}\right.$ from 
Ref. 31; $\phi_{255}=2.43 \mathrm{~cm}^{-1}$ from Ref. 32). The expressions relating nomenclature $C$ to nomenclature $\phi$ in $\mathrm{SF}_{6}$ have been given in Ref. 33 (Table 2, therein).

\section{EXPERIMENTAL}

\section{A. Description}

Scattering intensities were recorded from high purity (99.995\%) gaseous sulfur hexafluoride by using a conventional L-shaped Raman setup. ${ }^{26}$ A compact 532-nm continuous-wave diode-pumped solid-state frequencydoubled Nd:YV04 laser of a $2 \mathrm{~W}$ power was employed to shine a dice-large six-sided four-window high-pressure cell used to enclose the gaseous sample. A half-wave plate and a glan polarizer were put to make it possible to rotate the polarization of the incident beam from its initial direction, i.e., the direction vertical to the scattering plane $(\perp)$, to the parallel direction ( $\|)$. A double monochromator supplied with two 1800 grooves/mm holographic gratings was used to analyze the light scattered from the sample at $90^{\circ}$. Throughout the experiment the temperature of the sample was kept fixed at $294.5 \pm 1 \mathrm{~K}$. A nitrogen-cooled CCD was used for detection. Brut scattering signals (counts/s), denoted $A_{\perp}(v)$ and $A_{\|}(v)$, were recorded independently over a wide range of Raman frequency $v\left(\mathrm{~cm}^{-1}\right)$ for an incident beam polarization $\perp$ and $\|$ to the scattering plane, respectively. For each polarization of the incident beam, 13 independent experiments were run for 13 values of gas pressure, which were converted to density $\rho$ (in units of amagat) by means of an appropriate virial expansion; ${ }^{34}$ the lowest gas density used in the experiment amounted to 2.03 amagat, the highest one to 27.34 amagat.

\section{B. Calibration}

In order to calibrate on an absolute scale the recorded spectra, the Raman $S_{0}(1)$ rotational line of molecular hydrogen (centered at the frequency $v_{S_{0}(1)}=587.1 \mathrm{~cm}^{-1}$ ) was used as a signal of reference, and recorded at atmospheric pressure before and after each run. Key parameter for this procedure was the integrated scattering signal of $S_{0}(1)$, denoted $A_{i}^{i n t, H_{2}}$, with $i=\perp$ or $\|$. For every value of gas density, a formula involving $A_{i}^{i n t, H_{2}}$ ( $i=\perp$ or $\|$ ) was employed, ensuring the conversion of the recorded signals $A_{i}(v)$ to absolute intensities $I_{i}(v)$

$$
\begin{aligned}
I_{i}(v)= & \frac{2}{15} K_{i}(2 \pi)^{4}\left(v_{0}-v_{S_{0}(1)}\right)^{4} \gamma^{2} \\
& \times P_{J} \frac{3(J+1)(J+2)}{2(2 J+1)(2 J+3)} \frac{A_{i}(v)}{A_{i}^{\text {int } H_{2}}} \frac{n^{\prime}}{n} .
\end{aligned}
$$

In this formula, $K_{i}$ is a constant set to $\frac{7}{6}$ or 1 , depending on whether the experiment was held with a $\perp$ or with a \| polarization for the incident beam. Symbols $n$ and $n^{\prime}$ denote number density for $\mathrm{SF}_{6}$ and $\mathrm{H}_{2}$, respectively. Parameter $P_{J}$ is the fractional population of the $J$ th rotational level of $\mathrm{H}_{2}$ (here $J=1$ ) calculated upon considering Boltzmann statistics at the temperature of the experiment; the numerical value used for this parameter was taken from Ref. 35. The quantity $\gamma\left[=(3.18 \pm 0.03) \times 10^{-25} \mathrm{~cm}^{3}\right]^{36,37}$ denotes the anisotropy of $\mathrm{H}_{2}$ for the $S_{0}(1)$ line at the laser wavenumber $\left(v_{0}\right.$ $\left.=18797 \mathrm{~cm}^{-1}\right)$. Given that the only quantities in Eq. (1) to have dimensions are $\left(v_{0}-v_{S_{0}(1)}\right), \gamma, A_{i}(v)$, and $A_{i}^{i n t, H_{2}}$ (expressed in units of $\mathrm{cm}^{-1}, \mathrm{~cm}^{3}$, photons/s, and $\mathrm{cm}^{-1}$ photons/s, respectively), what Eq. (1) accomplishes is the passage from photons/s to a spectral intensity expressed in $\mathrm{cm}^{3}$.

\section{Processing: Removing the perturbing spectra}

To remove the perturbing neighboring spectra in the red and blue part of the overtone, a least-square fitting procedure was applied (twice per spectrum) to an exponential function $\exp \left[-\lambda\left(x-x_{0}\right)\right]$, with the two free parameters $x_{0}, \lambda$ optimized to fit the decreasing signal. We will see below that a simple plot of the recorded spectra along with the corresponding fit on a logarithmic scale, for all densities, is a particularly helpful device for illustrating the validity of this procedure. The slope $\lambda$ was found to be sensibly the same for all the values of $\rho$, leaving $x_{0}$ as the only changing parameter. A similar procedure was applied in the blue part of the spectrum. The two adjusted exponential functions, properly describing the shapes of the perturbing spectra at the left and the right of the $2 v_{5}$ overtone were calculated for each probed density and subtracted from the recorded spectrum. These parasitic spectra, whose origin was briefly discussed in our previous article, ${ }^{23}$ clearly distinguish themselves from the useful $2 v_{5}$ spectrum through the way their wings scale with density. This allowed us to successfully isolate the desired spectrum. In addition to the perturbing $\mathrm{SF}_{6}$ hot and combination bands, traces of residual hydrogen gas in the cell made their appearance in the recorded $\perp$ and $\|$ spectra of the overtone, by the occurrence, at the frequency $v_{S_{0}(3)}=1034.7 \mathrm{~cm}^{-1}$, of the fully depolarized rotational line $S_{0}(3)$. We removed from the recordings the portion of the spectrum around the frequency $1035 \mathrm{~cm}^{-1}$ where the contamination in the overtone from the $S_{0}(3)$ line was appreciable, and then we interpolated properly the empty strip in order to reliably access spectral moments.

In order to make the spectra recorded for various values of gas density clearly distinguishable on the same graph, in what follows, in addition to the two intensity quantities $I_{i}(v)$, use will be also made of the quantities $S_{i}(v)=I_{i}(v) . \rho$, with $i=\perp$ or $\|$. The latter are expressed in $\mathrm{cm}^{3}$ amagat.

\section{RESULTS}

\section{A. $\perp$ and || spectral components}

Figures 1(a) and 1(b) illustrate, as a function of frequency $v$, the experimental recordings $A_{\perp}(v) / A_{\perp}^{i n t, H_{2}}$ and $A_{\|}(\nu) / A_{\|}^{i n t, H_{2}}$ (empty symbols), respectively, for three values of gas density, along with the two adjusted exponential functions (solid-line curves) associated with each spectrum (see discussion above). The net overtone spectra obtained after removal of the perturbing neighboring bands are also shown for each density (filled symbols).

Figures 2(a) and 2(b) show, on a semilogarithmic scale, the $S_{\perp}$ and $S_{\|}$spectral components of the overtone, in units of 

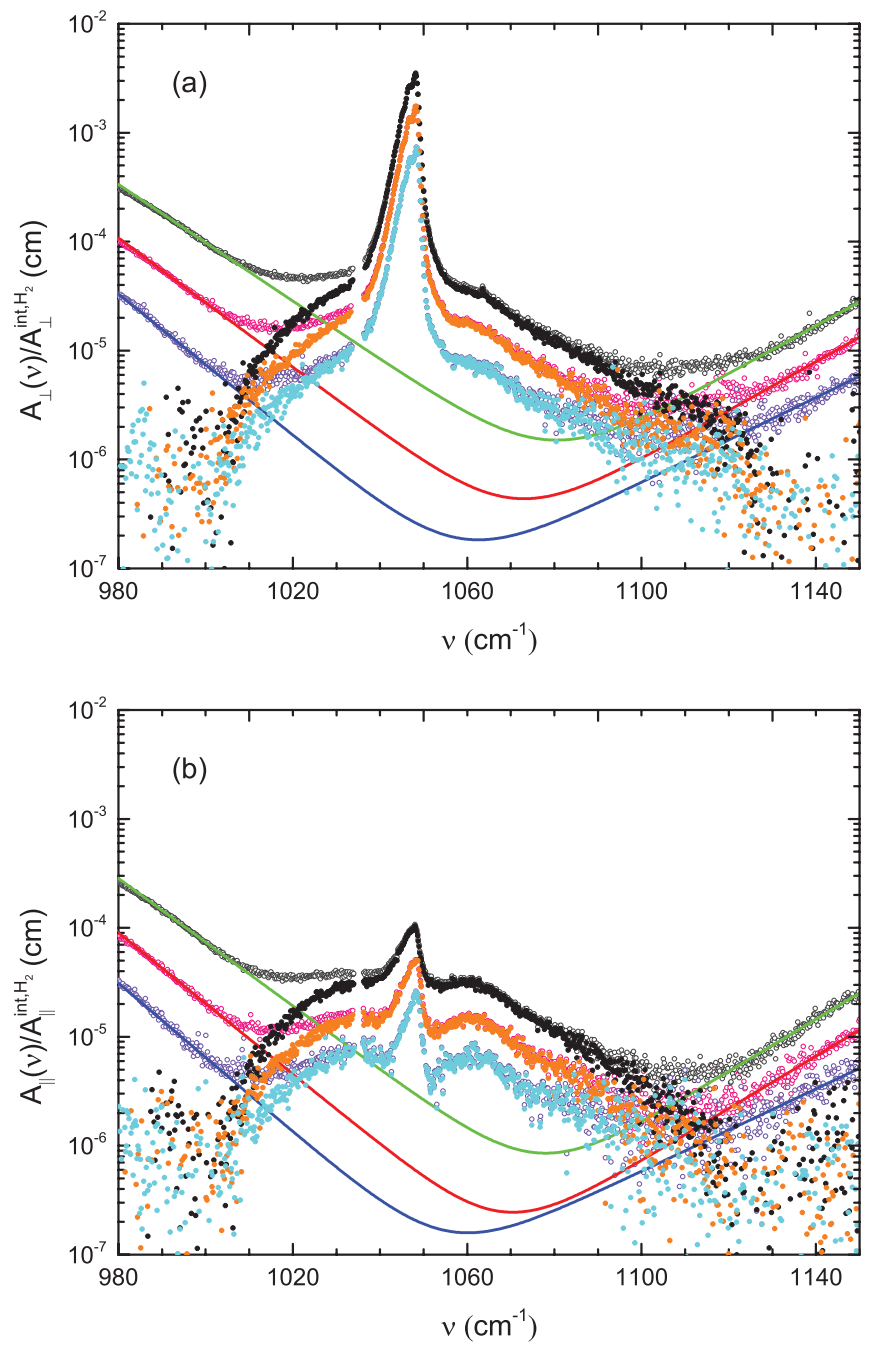

FIG. 1. (a) $\perp$ signal component recorded for three gas densities $(5.07,11.21$, and 21.83 amagat, in the upward direction) before (empty symbols) and after (filled symbols) extraction of parasitic signals that are due to the adjacent band wings. A careful fitting procedure was used to model the undesired baselines of these bands (solid line curves). (b) Same as (a) but for the || signal component.

$\mathrm{cm}^{3}$ amagat, for the 13 values of gas density and after removal of the baseline.

For each $\rho$, integration of $I_{i}(v)$ over $v$ was made, with as lower and upper bounds the values 1000 and $1110 \mathrm{~cm}^{-1}$, respectively. The result was found to be insensitive to the values of $\rho$, which is evidence of spectra strictly pertaining to isolated $\mathrm{SF}_{6}$ molecules, that is, of spectra that were reliably recorded, properly processed and analyzed, and successfully interpreted. Specifically, this observation offers compelling evidence that the protocol established for the extraction of the baseline was exact and that the obtained spectrum is indeed that of the sought overtone with no contamination by any binary, ternary, or higher-order collision-induced contributions or by parasitic effects due to other bands or compounds. Figure 3 showcases this property by illustrating the quantities $\sigma_{\perp}^{\prime}=\int S_{\perp}(v) d v$ (filled symbols) and $\sigma_{\|}^{\prime}=\int S_{\|}(v) d v$ (empty symbols) as a function of $\rho$. As expected, the symbols associated with each spectral component are tightly laid along a
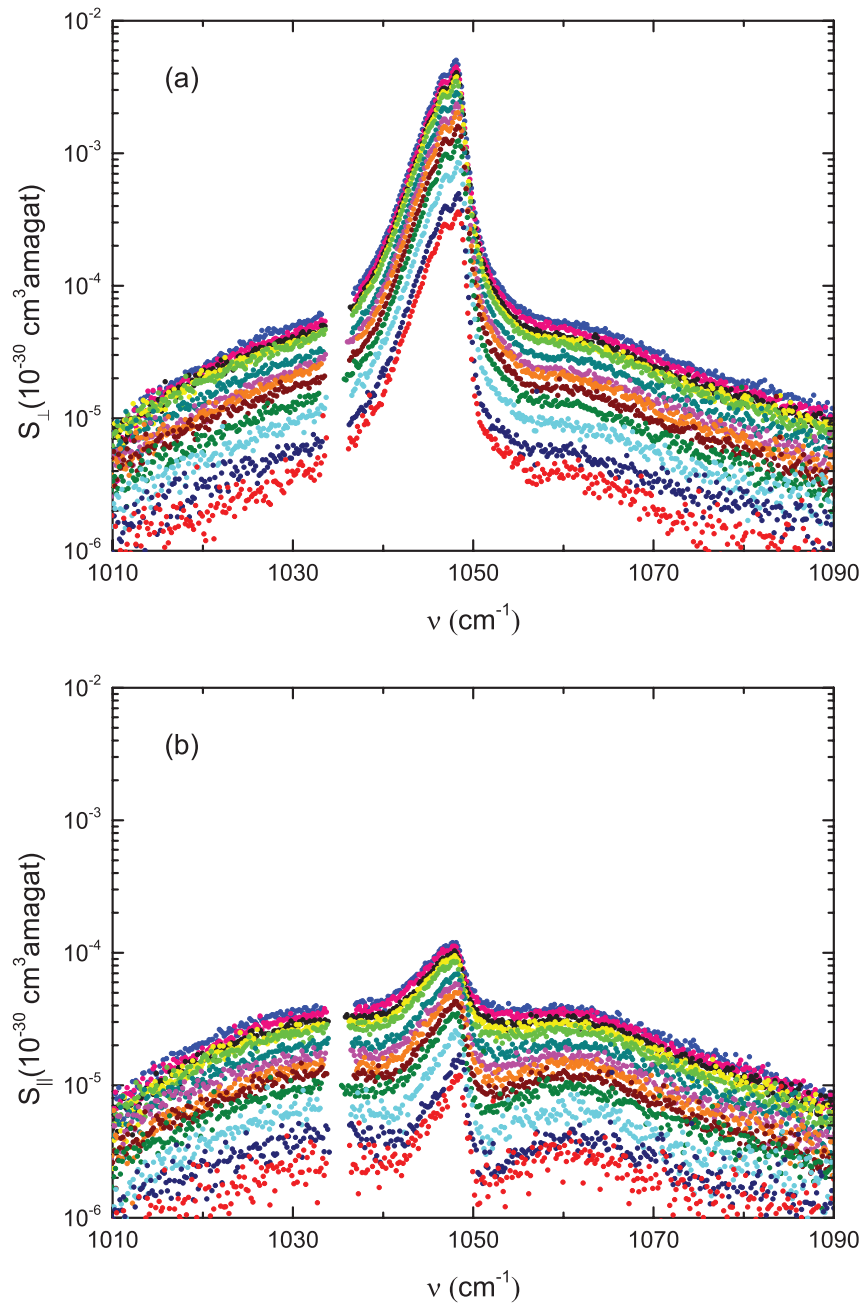

FIG. 2. (a) Absolute calibrated $\perp$ spectra (in $\mathrm{cm}^{3}$ amagat units) as a function of Raman frequency $v$ (in $\mathrm{cm}^{-1}$ units) for 13 values of $\rho$ ranging from 2 to 27 amagat (in the upward direction). (b) Same as (a) but for the \| spectra. For the sake of comparison with the $\perp$ spectra, same scale of axes was used as in (a).

straight line; for both spectra, the deviation of the symbols from their line fit was $<5 \%$.

\section{B. Anisotropic spectrum, depolarization ratio, and the two lower-order classical moments}

Anisotropic, $I_{a n i}(v)$, and isotropic, $I_{i s o}(v)$, spectra were deduced from $I_{\perp}(v)$ and $I_{\|}(v)$, for each of the 13 values of $\rho$, by means of two linear transformations that take into account the aperture of the scattered beam. $I_{a n i}(v)$ is the only spectrum with which we are concerned in this article. Thorough analysis of the far stronger isotropic component of the $2 v_{5}$ overtone has been reported in our preceding article ${ }^{23}$ any mention of $I_{i s o}(v)$ will be skipped below, with the exception of a few places in the text where numerical information related to the isotropic spectrum is given for comparison purposes.

The transformation for $I_{a n i}(v)$ reads ${ }^{11}$

$$
I_{a n i}(v)=1.01 \cdot I_{\|}(v)-0.01009 \cdot I_{\perp}(v) .
$$




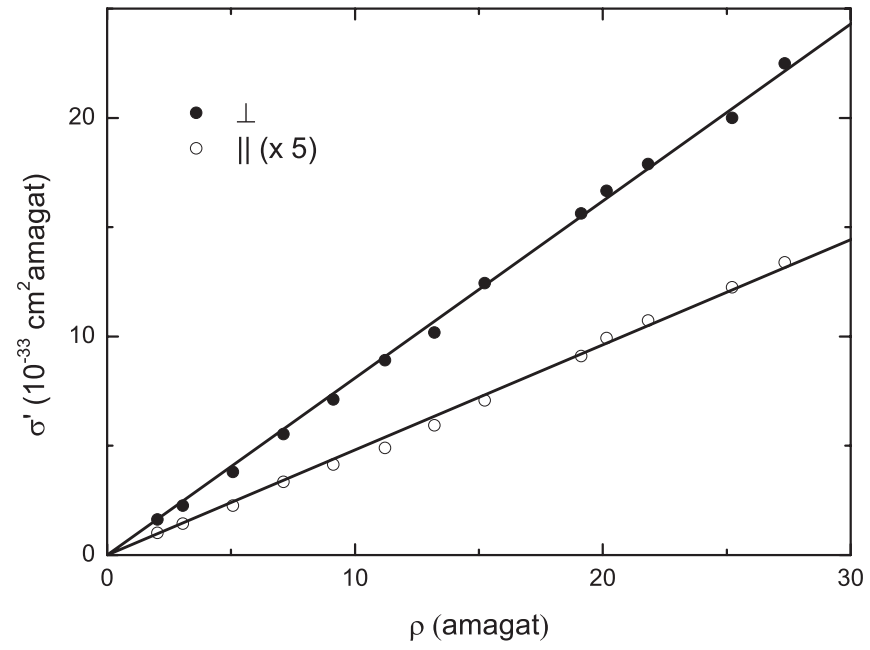

FIG. 3. Integrated intensity $\sigma^{\prime}=\int S(v) d v$ (in $\mathrm{cm}^{2}$ amagat units) as a function of density $\rho$ (in amagat units) for the two spectral components $S_{\perp}(\bullet)$ and $S_{\|}$(०). For the sake of clarity, the integrated intensities of $S_{\|}$were scaled by a factor 5 . Both series of symbols ( $\bullet$ and $\circ$ ) are seen to lay along straight lines passing from zero. The property that each spectrum has a unique slope reveals that its integrated intensity does not depend on $\rho$, and hence the zeroth-order moment does not depend either. The fact that at zero density the signal is absent is evidence for the absence of noise background.

Figure 4 shows as a function of $v\left(\right.$ in $\left.\mathrm{cm}^{-1}\right)$ the intensity $I_{a n i}$ of the anisotropic overtone spectrum (in units of $\mathrm{cm}^{3}$ ) for the 13 values of gas density ranging from 2 to 27 amagat. Same associations as the ones used in the preceding figures as well as in those of Ref. 23 are made between the values of density and the colors depicting the spectra. A $Q$ branch centered at $1048 \mathrm{~cm}^{-1}$ is clearly discernible on the figure, along with $O$ and $S$ branches that appear equally well. Recall that, in the case of the isotropic spectrum, there was solely a single branch present on the figure (Figure 6 in Ref. 23). The underlying rotational structure remains unresolved. A procedure, whose technical details are described at the end of Subsection V C, permitted to reveal a red shift for the $Q$ branch, along with a slight increase of its integrated intensity, amounting to $-5(2) \times 10^{-2} \mathrm{~cm}^{-1}$ amagat $^{-1}$ and $(1.444$ $+0.028 \rho) \times 10^{-35} \mathrm{~cm}^{2}$, respectively. This is illustrated in the right and left inset of the figure.

To quantify the level of polarization of the signal, the depolarization ratio $\eta(v)$ is a useful property defined by the quotient

$$
\eta(v)=\frac{I_{\|}(v)}{I_{\perp}(v)} .
$$

An integrated depolarization ratio can also be defined by

$$
\eta_{i n t}=\frac{\int I_{\|}(\nu) d v}{\int I_{\perp}(v) d v} .
$$

Figure 5 illustrates the depolarization ratio $\eta(v)$ of the overtone as a function of Raman frequency $v$, for three representative values of gas density. As inferred from the figure, the overtone is highly polarized around $1048 \mathrm{~cm}^{-1}$, as a consequence of the fact that, at the top of the $Q$ branch, the $S_{\perp}$

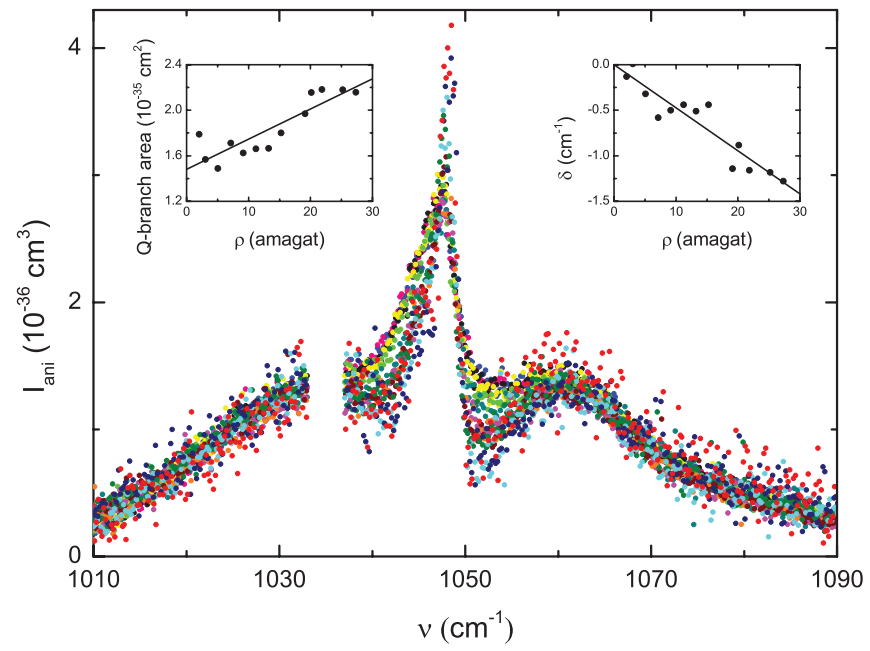

FIG. 4. $I_{\text {ani }}\left(\right.$ in $\mathrm{cm}^{3}$ ) as a function of $v\left(\right.$ in $\mathrm{cm}^{-1}$ ) for 13 values of $\rho$ ranging from 2 to 27 amagat. Same associations as the ones made above are to be made between the values of $\rho$ and the colors depicting the spectra. The $O, Q$, and $S$ branches are clearly seen. The rotational structure remains unresolved. The left and right inset illustrate the way in which integrated intensity and position of the $Q$-branch, respectively, are affected by the gas density (see text, Subsection V C).

spectra are 50 times more intense than their $S_{\|}$counterparts (see Figures 2(a) and 2(b)). In contrast, the depolarization ratio at the edges attains its maximum value, $\frac{6}{7}$. Equally interesting is the fact that, for all $\rho$ values, the shape of $\eta(v)$ remains unchanged, a property suggesting that the function $\eta(v)$ must preserve its shape even at the zero-density limit.

As for the integrated depolarization ratio, application of Eq. (4) results in the very small value $\eta_{\text {int }}=0.12(1)$, a property demonstrating the highly polarized character of the overtone. This value is in agreement with the value $\eta_{\text {int }}$ $\approx 0.10$, which results from the estimate $\eta_{s} \approx 0.05$ previously reported by Holzer and Ouillon ${ }^{21}$ upon appropriate

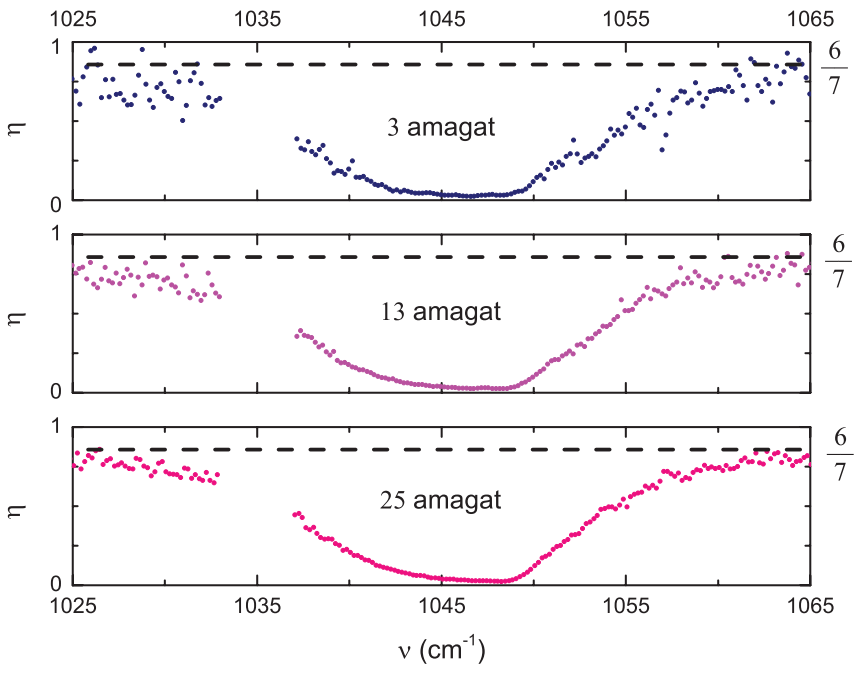

FIG. 5. Depolarization ratio $\eta(v)$ as a function of $v\left(\right.$ in $\left.\mathrm{cm}^{-1}\right)$ for three representative gas density values, $\rho=3,13$, and 25 amagat. The overtone is highly polarized in the middle of the spectrum and depolarized at the edges. The dashed lines indicate the theoretical upper bound of $\eta$. The fact that the shape of $\eta(v)$ remains unchanged in the three panels suggests that a similar shape should be expected in the zero-density limit. 
TABLE I. Anisotropic and isotropic zeroth-order $\left(M_{0}\right)$, second-order $\left(M_{2}\right)$, and reduced $\left(\bar{M}_{2}=\frac{M_{2}}{M_{0}}\right)$ spectral moments. $M_{0}$ is given in $\times 10^{-54} \mathrm{~cm}^{6}$, $M_{2}$ in $\times 10^{-30} \mathrm{~cm}^{6} \mathrm{~s}^{-2}$, and $\bar{M}_{2}$ in $\times 10^{23} \mathrm{~s}^{-2}$. Whenever assuming Gaussian bandshapes, reduced moments allow to assess, via the expression $\Gamma / 2=(2 \pi c)^{-1} \sqrt{\bar{M}_{2}}$, band halfwidths at $1 / e^{\frac{1}{2}}$-maximum. Within this assumption, the bandwidth amounts to $22.4 \mathrm{~cm}^{-1}$ for the anisotropic spectrum and to $2.4 \mathrm{~cm}^{-1}$ for the isotropic spectrum.

\begin{tabular}{lcc}
\hline \hline Spectral moment & Anisotropic & Isotropic \\
\hline$M_{0}$ & $4.31(65)$ & $4.59(69)$ \\
$M_{2}$ & $77(15)$ & $0.98(20)$ \\
$\bar{M}_{2}$ & 180 & 2.1 \\
\hline \hline
\end{tabular}

conversion of the concept issuing from the definition $\eta_{s}$ $=\int I^{(\perp \|)}(v) d \nu / \int I^{(\perp \perp)}(\nu) d v$ to that defined through Eq. (4). Recall that the two integrated properties are related with each other through the expression $\eta_{\text {int }}=\frac{2 \eta_{s}}{1+\eta_{s}}$ and that their upper bounds are different: $\eta_{s}^{\max }=\frac{3}{4}, \eta_{\text {int }}^{\max }=\frac{6}{7}$.

Finally, zeroth-order and second-order classical spectral moments, $M_{2 n}(n=0,1)$, can be defined for the anisotropic spectrum through the following formula:

$$
\begin{aligned}
M_{2 n}= & \frac{15}{2}(2 \pi c)^{2 n}(2 \pi)^{-4}\left(v_{0}-v_{s}\right)^{-4} \\
& \times \int I_{a n i}(v)\left(v-v_{s}\right)^{2 n} d \nu .
\end{aligned}
$$

In this expression, the $\frac{15}{2}$ factor characterizes anisotropic spectra and $v_{s}=1048 \mathrm{~cm}^{-1}$ is the wavenumber at the center of the overtone.

Table I shows the values of the anisotropic zeroth-order, second-order, and reduced second-order $\left(\bar{M}_{2}=\frac{M_{2}}{M_{0}}\right)$ classical spectral moments, in units of $10^{-54} \mathrm{~cm}^{6}, 10^{-30} \mathrm{~cm}^{6} \mathrm{~s}^{-2}$, and $10^{23} \mathrm{~s}^{-2}$, respectively, as they were calculated by using Eq. (5). For the sake of comparison, values of the same quantities are also shown for the isotropic spectrum. Note that for Gaussian bandshapes, the reduced moment $\bar{M}_{2}$ allows to assess, via the expression $(2 \pi c)^{-1} \sqrt{\bar{M}_{2}}$, band halfwidths at $1 / e^{\frac{1}{2}}$-maximum. Within this assumption, the bandwidth of the overtone amounts to $22.4 \mathrm{~cm}^{-1}$ for the anisotropic spectrum; for the far narrower isotropic spectrum, it amounts to only $2.4 \mathrm{~cm}^{-1}$.

\section{Bandshape and width, pressure broadening and shift}

A Gaussian function was used to interpolate the left and right wings of the measured profile. Figure 6 illustrates the result for a representative value of gas density. The value of the integrated intensity obtained from the interpolated profile was found to closely approach the one obtained from direct integration of the experimental data. (The deviation between the experimental data and the model curve returned by the fitting procedure, in the frequency domain $1000-1110 \mathrm{~cm}^{-1}$, was slight, with fluctuations going from $0.8 \%$ to $2.5 \%$ and with a mean value of $1.3 \%$.) Furthermore, a narrowing of the Gaussian was observed with increasing gas density, illustrated in the right inset of the figure. This illustration reveals that the dependence of the halfwidth on the gas density is linear,

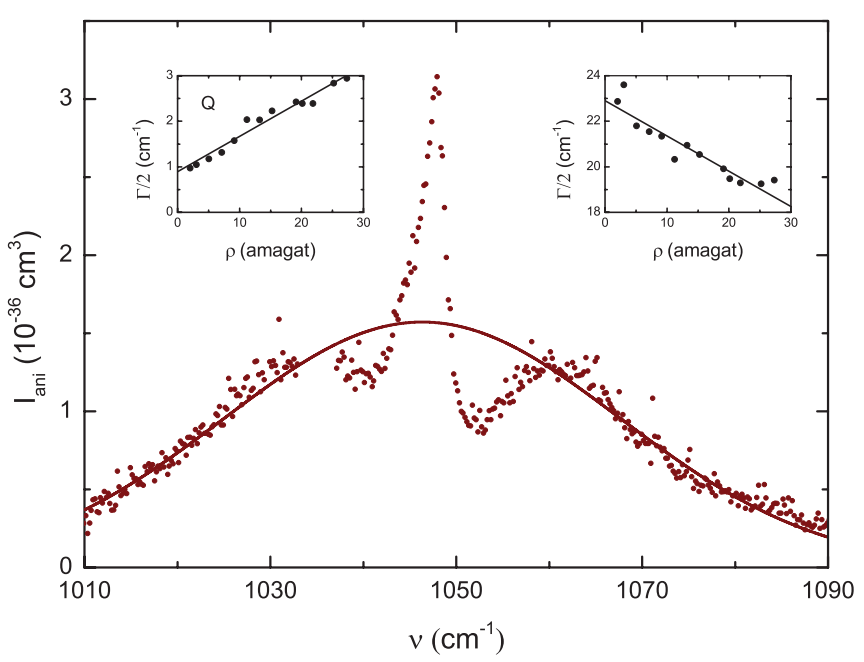

FIG. 6. To showcase the procedure that we followed to reveal any pressure broadening or narrowing effects on the anisotropic spectrum, the spectrum is shown (symbols) for a representative density value $(\rho=9.13$ amagat) along with a Gauss-shaped function (solid line curve) that best interpolates the left and right wings of the measured profile. Our study revealed pressure broadening of the $Q$ branch (left inset) but narrowing of the whole spectrum (right inset), both scaling linearly with $\rho$.

a fact allowing us to deduce the narrowing coefficient of the spectrum $\left(\gamma \simeq-0.155 \mathrm{~cm}^{-1}\right.$ amagat $\left.^{-1}\right)$ along with its zerodensity halfwidth $\left(\Gamma / 2=22.9(6) \mathrm{cm}^{-1}\right)$. It is gratifying that the zero-density halfwidth measured in this way is in excellent agreement with the value $22.4 \mathrm{~cm}^{-1}$ obtained from the expression $(2 \pi c)^{-1} \sqrt{\bar{M}_{2}}$ (see Table I).

In the effort to understand also the way in which the $Q$ branch behaves on its own, the anisotropic spectrum was first analyzed for an intermediate value of gas density, as a compromise between spectral resolution and signal strength, and the converged procedure was then applied to the other values of $\rho$. Specifically, adjustment of the $O$ and $S$ branches to appropriate model-shapes was made, for that intermediate value of $\rho$, by using standard gamma distribution. ${ }^{38}$ The modeled branches were then removed from the total spectrum to make the entire body of the $Q$ branch appear. This procedure was repeated for all values of $\rho$, by using the optimal profile described above. Unlike the narrowing of the anisotropic spectrum as a whole, the described analysis showed a broadening of the $Q$ branch with increasing gas density. This is illustrated in the left inset of Figure 6. From this illustration, a linear dependence is once again revealed, a fact allowing us to derive the broadening coefficient $\left(\gamma \simeq 0.078(5) \mathrm{cm}^{-1}\right.$ amagat $\left.^{-1}\right)$ and the zero-density halfwidth $\left(\Gamma / 2=0.89(9) \mathrm{cm}^{-1}\right)$ of the $Q$ branch. As anticipated in Subsection V B and illustrated in the

TABLE II. Band halfwidths $\left(\frac{\Gamma}{2}\right)$ and shifts $(\delta)$, in $\mathrm{cm}^{-1}$, along with the density dependence of these properties for the overtone anisotropic spectrum and for its $Q$ branch alone. The same quantities are also shown for the isotropic spectrum.

\begin{tabular}{lccc}
\hline \hline & $\begin{array}{c}\text { Anisotropic } \\
(Q \text {-branch only })\end{array}$ & $\begin{array}{c}\text { Anisotropic } \\
\text { (entire) }\end{array}$ & Isotropic \\
\hline$\frac{\Gamma}{2}\left(\mathrm{~cm}^{-1}\right)$ & $0.89+0.078 \rho$ & $22.93-0.155 \rho$ & $1.3575-0.0008 \rho$ \\
$\delta\left(\mathrm{cm}^{-1}\right)$ & $-0.05 \rho$ & & $-0.01 \rho$ \\
\hline
\end{tabular}


TABLE III. Absolute values of transition matrix-elements $(000020) \leftarrow(000000)$ of the $2 v_{5}$ polarizability anisotropy $\Delta \alpha$ along with values for the upper bounds of the matrix-elements of the two experimentally indistinguishable anisotropy components $\left(\alpha_{x x}-\alpha_{y y}\right)$ and $\alpha_{x y}$. For the sake of completeness, the corresponding values of the mean polarizability $\bar{\alpha}$ are also given from our and previous studies. All values are in $\mathrm{cm}^{3}$. The symmetry species for each polarizability component is also indicated.

\begin{tabular}{|c|c|c|c|}
\hline$|\langle 000020|\Delta \alpha| 000000\rangle|$ & $\left|\left\langle 000020\left|\alpha_{x x}-\alpha_{y y}\right| 000000\right\rangle\right|$ & $\left|\left\langle 000020\left|\alpha_{x y}\right| 000000\right\rangle\right|$ & $|\langle 000020|\bar{\alpha}| 000000\rangle|$ \\
\hline$E_{g}, F_{2 g}$ & $E_{g}$ & $F_{2 g}$ & $A_{1 g}$ \\
\hline $1.92(14) \times 10^{-27}$ & $1.6 \times 10^{-27}$ & $0.6 \times 10^{-27}$ & $\begin{aligned} 1.98(14) & \times 10^{-27} \\
1.95 & \times 10^{-27 \mathrm{a}} \\
1.58 & \times 10^{-27 \mathrm{~b}}\end{aligned}$ \\
\hline
\end{tabular}

${ }^{\mathrm{a}}$ Shelton and Ulivi, Ref. 22

${ }^{\mathrm{b}}$ Holzer and Ouillon, Ref. 21.

right and left inset of Figure 4, this procedure permits also to reveal a red shift for the $Q$ branch, along with a slight increase of its integrated intensity.

Table II gathers halfwidths, shifts, and pressure broadening or narrowing laws for the overtone anisotropic spectrum and for its $Q$ branch alone. The same properties for the isotropic spectrum of the overtone are also shown, allowing for a comparative analysis between the two spectral components.

\section{The matrix-elements $(\Delta \alpha)_{f i}$ and $(\bar{\alpha})_{f i}$}

In view of the results obtained in Sec. V for the zerothorder moment and of the discussion made in Sec. III, absolute values of transition matrix-elements $(000020) \leftarrow(000000)$ for the polarizability anisotropy $\Delta \alpha$ along with values for the upper bounds of the matrix-elements of the two experimentally indistinguishable anisotropy components $\left(\alpha_{x x}-\alpha_{y y}\right)$ and $\alpha_{x y}$ can now be derived. These are gathered in Table III. The corresponding values for the mean polarizability $\bar{\alpha}$ are also given for the sake of completeness.

\section{SYNOPSIS}

In this article, we show the existence of an anisotropic Raman component for the first "scissoring" overtone of sulfur hexafluoride and report the first observation and analysis of its spectrum at room temperature. This overtone has been subject of fragmentary studies in the 1970s and the 1980s, which revealed a weak and narrow isotropic spectrum and an extremely small depolarization ratio. At that time, sensitivity was not an issue and little could lead anyone to predict what Raman instrumentation would accomplish in the years to come. The high sensitivity of our experiment along with the strategy and the demanding protocol we follow for signal detection and processing have now made it possible to measure this very weak anisotropic component, and to reveal a multitude of properties related to its $O-Q-S$ structure and to its isolated $Q$ branch: pressure broadening and narrowing, shifts, widths, intensities. The anisotropic spectrum was found to be about ten times broader than its isotropic counterpart and to peak about 50 times below the latter component, yet the zeroth-order moments in the two spectra were found to take near equal values. This finding is evidence of a transition $f \leftarrow i$ with matrix-elements $(\Delta \alpha)_{f i}$ and $(\bar{\alpha})_{f i}$ taking large and near equal absolute values, and rules out the mainstream naïve view that a weak anisotropic Raman spectrum automatically means a small value for the transition anisotropy. Given the systematic and stiff protocol of our analysis, we believe that the whole range of spectral lines, regardless of whether they are fundamentals, overtones, or combination bands, deserve to be reexamined in $\mathrm{SF}_{6}$, toward a complete description of dipole moment and polarizability properties.

\section{ACKNOWLEDGMENTS}

We are grateful to Dr. A. P. Kouzov for fruitful discussions. One of us (D.K.) gratefully acknowledges doctoral fellowship support from the CNRS and "la Région des Pays de la Loire."

${ }^{1}$ D. A. Long, The Raman Effect: A Unified Treatment of the Theory of Raman Scattering by Molecules (Wiley, Chichester, UK, 2002).

${ }^{2}$ G. C. Tabisz, in Molecular Spectroscopy (A Specialist Periodical Report), edited by R. F. Barrow, D. A. Long, and J. Sheridan, (Chemical Society, London, 1979), Vol. 6, pp. 136-173.

${ }^{3}$ E. B. Wilson, Jr., J. C. Decius, and P. C. Cross, Molecular Vibrations: The Theory of Infrared and Raman Vibrational Spectra (Dover, New York, 1980).

${ }^{4}$ L. Frommhold, Adv. Chem. Phys. 46, 1 (2007).

${ }^{5}$ Collision- and Interaction-Induced Spectroscopy, edited by G. C. Tabisz and M. N. Neuman (Kluwer, Dordrecht, 1995).

${ }^{6}$ T. Bancewicz, Y. Le Duff, and J.-L. Godet, Adv. Chem. Phys. 119, 267 (2002).

${ }^{7}$ J.-M. Hartmann, C. Boulet, and D. Robert, Collisional Effects on Molecular Spectra: Laboratory Experiments and Model, Consequences for Applications (Elsevier, Amsterdam, 2008).

${ }^{8}$ I. A. Verzhbitskiy, M. Chrysos, F. Rachet, and A. P. Kouzov, Phys. Rev. A 81, 012702 (2010).

${ }^{9}$ M. Chrysos and I. A. Verzhbitskiy, Phys. Rev. A 81, 042705 (2010).

${ }^{10}$ I. A. Verzhbitskiy, M. Chrysos, and A. P. Kouzov, Phys. Rev. A 82, 052701 (2010).

${ }^{11}$ M. Chrysos, I. A. Verzhbitskiy, F. Rachet, and A. P. Kouzov, J. Chem. Phys. 134, 044318 (2011).

${ }^{12}$ M. Chrysos, I. A. Verzhbitskiy, F. Rachet, and A. P. Kouzov, J. Chem. Phys. 134, 104310 (2011).

${ }^{13}$ I. A. Verzhbitskiy, A. P. Kouzov, F. Rachet, and M. Chrysos, J. Chem. Phys. 134, 194305 (2011)

${ }^{14}$ I. A. Verzhbitskiy, A. P. Kouzov, F. Rachet, and M. Chrysos, J. Chem. Phys. 134, 224301 (2011).

${ }^{15}$ S. M. El-Sheikh, G. C. Tabisz, and R. T. Pack, J. Chem. Phys. 92, 4234 (1990).

${ }^{16}$ G. Maroulis, Chem. Phys. Lett. 312, 255 (1999).

${ }^{17}$ T. Bancewicz, J.-L. Godet, and G. Maroulis, J. Chem. Phys. 115, 8547 (2001).

${ }^{18}$ J.-L. Godet, F. Rachet, Y. Le Duff, K. Nowicka, and T. Bancewicz, J. Chem. Phys. 116, 5337 (2002).

${ }^{19}$ Y. Le Duff, J.-L. Godet, T. Bancewicz, and K. Nowicka, J. Chem. Phys. 118, 11009 (2003).

${ }^{20}$ If the Cartesian coordinates of the $X Y_{6}$ atoms are $X(0,0,0), Y_{1}(0,0, R)$, $Y_{2}(0,0,-R), Y_{3}(R, 0,0), Y_{4}(-R, 0,0), Y_{5}(0, R, 0)$, and $Y_{6}(0,-R, 0)$, and 
$R$ is the extension of the bond $X-Y$ at equilibrium, then the symmetry coordinate of the $\nu_{5}$ vibration reads $\frac{1}{2} R\left(\alpha_{35}-\alpha_{36}-\alpha_{45}+\alpha_{46}\right)$, where $\alpha_{a b}$ is the angle between the bonds $X-Y_{a}$ and $X-Y_{b}$.

${ }^{21}$ W. Holzer and R. Ouillon, Chem. Phys. Lett. 24, 589 (1974).

${ }^{22}$ D. P. Shelton and L. Ulivi, J. Chem. Phys. 89, 149 (1988).

${ }^{23}$ D. Kremer, F. Rachet, and M. Chrysos, J. Chem. Phys. 138, 174308 (2013).

${ }^{24}$ The meaning of the two double-superscript quantities $I^{(\perp \|)}$ and $I^{(\perp \perp)}$ is scattering intensity recorded at the output when the incident beam is polarized perpendicular $(\perp)$ to the scattering plane (first superscript) and the output beam analyzed in the direction parallel $(\|)$ and perpendicular $(\perp)$ to the scattering plane, respectively (second superscript).

${ }^{25}$ F. Rachet, M. Chrysos, C. Guillot-Noël, and Y. Le Duff, Phys. Rev. Lett. 84, 2120 (2000).

${ }^{26}$ F. Rachet, Y. Le Duff, C. Guillot-Noël, and M. Chrysos, Phys. Rev. A 61, 062501 (2000).

${ }^{27}$ Y. Le Duff, Phys. Rev. Lett. 90, 193001 (2003).

${ }^{28}$ M. Chrysos, A. P. Kouzov, N. I. Egorova, and F. Rachet, Phys. Rev. Lett. 100, 133007 (2008).

${ }^{29}$ S. Dixneuf, M. Chrysos, and F. Rachet, J. Chem. Phys. 131, 074304 (2009).

${ }^{30}$ Confusion between symbols $k$ and $\phi$, often used indifferently to denote cubic force constants, is regularly source of errors in matrix-element calculations and of misunderstandings regarding the real physical significance of these constants; in this context, see, for instance, a recent comment by M. Chrysos and D. Kremer, Int. J. Quantum Chem. 113, 2634 (2013).

${ }^{31}$ B. J. Krohn and J. Overend, J. Phys. Chem. 88, 564 (1984).

${ }^{32}$ D. P. Hodgkinson, J. C. Barrett, and A. G. Robiette, Mol. Phys. 54, 927 (1985).

${ }^{33}$ D. P. Hodgkinson, R. K. Heenan, A. R. Hoy, and A. G. Robiette, Mol. Phys. 48, 193 (1983).

${ }^{34}$ J. J. Hurley, D. R. Defibaugh, and M. R. Moldover, Int. J. Thermophys. 21, 739 (2000).

${ }^{35}$ B. P. Stoicheff, Can. J. Phys. 35, 730 (1957).

${ }^{36}$ J. Rychlewski, J. Chem. Phys. 78, 7252 (1983).

${ }^{37}$ C. Schwartz and R. J. Le Roy, J. Mol. Spectrosc. 121, 420 (1987).

${ }^{38}$ The chosen function was given the form $g(v)=H\left[\beta\left(v-v_{s}\right)\right] C G(v)$, with $H$ the Heaviside step function, $C$ and $\beta$ free parameters, and $G(v)$ $=\frac{\beta^{\alpha}\left(v-v_{s}\right)^{\alpha-1}}{(\alpha-1) !} \exp \left[-\beta\left(v-v_{s}\right)\right]$ the unity-normalized shape function for a gamma distribution probability density with random variable $v-v_{s}$, shape $\alpha(=2)$ and rate $\beta$. This function, whose choice was dictated by probability theory, turned out to faithfully reproduce the exponential decrease of the $O$ and $S$ branches and their rise from the origin $\left(v=v_{s}\right)$. The converged $M_{0}$ values for the branches $O$ and $S$ are $1.71 \times 10^{-54} \mathrm{~cm}^{6}$ and $1.61 \times 10^{-54} \mathrm{~cm}^{6}$, respectively. 